\title{
Erratum
}

\section{Radiative corrections set up inflation: dynamical models}

Saul Barshay, Georg Kreyerhoff

Z. Phys. C 75 (1997) 167

As Fig. 8, corresponding to the "note added in proof", the previous Fig. 6 has been erroneously reproduced. The correct Fig. 8 is printed here.

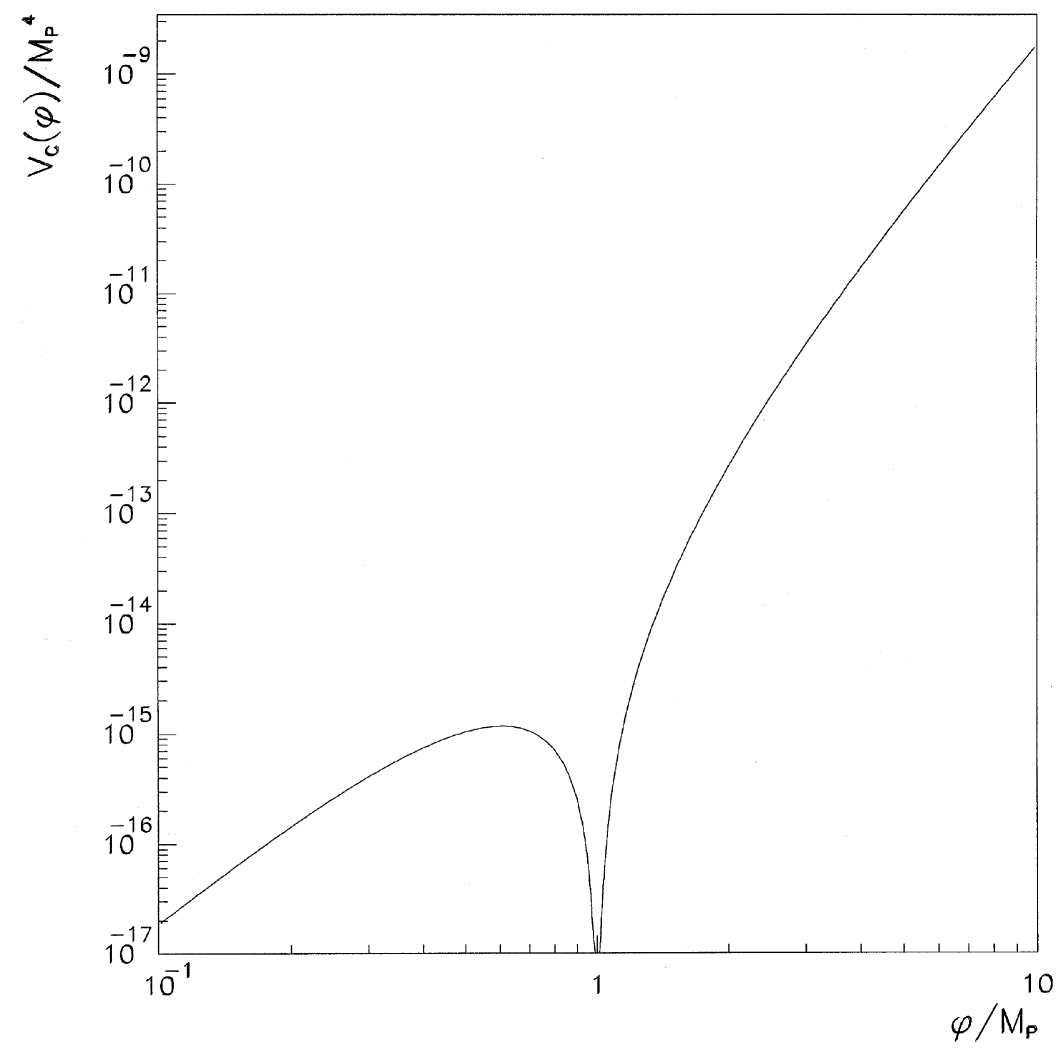

Fig. 8. The radiatively-corrected tree-level potential $V_{c}(\phi)=\lambda(\phi) \phi^{4}$ for the "inverted" situation described in the "note added in proof" 